\title{
Fluid and Nutritional Management Can Significantly Reduce the Mortality of Patients with Eclampsia in Resource Poor Settings
}

\author{
BADRUNNESA BEGUM ${ }^{1}$, NASREEN AKHTER ${ }^{2}$, MD. KAMAL UDDIN ${ }^{3}$, MD. ABDULAZIZ $^{4}$, \\ KASHFIA KAMAL NOVA ${ }^{5}$
}

\begin{abstract}
:
Objective: To observe fluid and nutritional management along with specific management and close clinical monitoring without intensive care management improve the condition of unconcious eclamptic patients in the resource poor setting.

Method: Six hundred and nine(609) eclamptic patients were admitted in Mymensingh Medical College Hospital from January to December 2008. Twenty four (24) unconscious eclamptic patients were enrolled for this clinical trial study. These patients were managed in eclampsia ward with fluid therapy of $0.9 \%$ sodium chloride, $25 \%$ glucose, $5 \%$ aminoacids along with hydrocortisone and in some cases nasogastric feeding in addition to other regular medications. They were compared with 26 eclampsia patients treated with normal regular hospital management. A systematic guideline was followed and patients were closely monitored until the outcome.
\end{abstract}

Results: The mean age of 23 years, 18 cases were primi-gravida; 16 had intrapartum, 6 had postpartum and 2 had antepartum eclampsia. The mean number of convulsion before admission was 12. The mean Glasgow Coma Scale (GCS) of these patients during admission was 5, which improved to 10.3 in 24 hours and 14.5 in 36 hours. None of these patients had fatal outcome in compare to observation group where three patients died following complications.

Conclusion: Close monitoring with fluid and nutritional management can significantly reduce the mortality of unconscious eclamptic patients in resource poor settings where intensive care facility is limited. Development and adaptation of feasible systematic guideline for the management of unconscious eclamptic patient should be scaled up for the resource poor settings of developing and under developed countries.

Keywords: Unconcious eclamptic patient, fluid and nutrition therapy, resource poor setting.

Introduction:

Eclampsia is the occurrence of seizure in the presence of pre eclampsia (PE). It is normally thought to be triggered by severe cerebral vasospasm, haemorrhage, ischaemia or oedema. It is a leading cause of material death (16 to $18 \%$ ) in Bangladesh. Fluid management is important in severe PE because of the low plasma volume and cardiac output which increase the likelihood of fetal distress and oliguria, particularly after vasodilatation. However, endothelial damage, low oncotic pressure and excessive fluid administration increase the risk of pulmonary oedema ${ }^{1}$. Plasma volume depletion is the cause of hypertension in pre-eclampsia and alternatively the hypertension is secondary to a contracted vascular bed which

1. Asst. Prof. Gynae, Khulna Medical College

2. Asst. Prof. Gynae, Khulna Medical College

3. Asst. Prof. Pharmacology, Khulna Medical College

4. Intern Doctor, Khulna Medical College Hospital

5. $5^{\text {th }}$ year MBBS student, Uttara Adunik Medical College, Dhaka 
results in impaired utero-placental perfusion ${ }^{2}$. Volume expansion and reduction in blood pressure have been attempted with and without central haemodynamic monitoring.

\section{Materials and Methods:}

A clinical trial was done in one of the largest tertiary hospital. Six hundred and nine (609) eclamptic patients were admitted in Mymensingh Medical College Hospital from January to December 2008. We enrolled 24 unconscious eclamptic patients having Glassgo Coma Scale (GCS) from 3 to 10 taking Ethical clearance from the institution admitted under the principal investigator. Informed written consent was taken from patient attendants. Comparative observation study was done among 26 patients having same score but they were treated with national standard protocol. That was accessed with Hartman's solution 1 litre, $5 \%$ dextrose in aqua $500 \mathrm{ml}$ and $5 \%$ dextrose in saline $500 \mathrm{ml}$ total 2 litres of fluid plus last 24 hours output with strict fluid balance maintaining intake and output chart.

In eclampsia ward, intervention was done with $0.9 \%$ sodium chloride 1 liter, $25 \%$ glucose 200 cc 12 hourly,
5\% amino-acids 1 liter around 24 hours along with hydrocortisone and in some cases nasogastric feedings in addition to other regular medications such as antihypertensive, anticonvulsant etc. We followed a systematic guideline and closely monitored those patients until the outcome.

\section{Observation and Results:}

The mean age of 23 years, 18 cases were primigravida; 16 had intrapartum, 6 had postpartum and 2 had antepartum eclampsia. The mean number of convulsion before admission was 12 . The mean GCS of these patients during admission was 5 , which was improved to 10.3 in 24 hours and 14.5 in 36 hours. None of these patients had fatal outcome in compare to three deaths among the 26 observation group of total 609 admitted patients

Table-1 showing different clinical findings with time.

Fig.-1 showing graphical presentation of the improvement of the patients in terms of diminuation of number of convulsion after intervention with fluid and nutrition therapy along with standard antihypertensive and anti convulsant therapy.

Table-I

\begin{tabular}{lcccc}
\hline Time & $\begin{array}{c}\text { Mean Glasgow } \\
\text { coma scale }\end{array}$ & $\begin{array}{c}\text { Mean number } \\
\text { of Convulsion }\end{array}$ & $\begin{array}{c}\text { Mean Blood } \\
\text { pressure (mm of Hg) }\end{array}$ & $\begin{array}{c}\text { Mean Proteinuria } \\
\text { (gm/day) }\end{array}$ \\
\hline At Admission(0 time) & 5 & $>14$ & $170 / 110$ & 13 \\
At 24 hours & 10.3 & 4 & $160 / 100$ & 9 \\
At 36 hours & 14.5 & $<2$ & $140 / 95$ & 2 \\
\hline
\end{tabular}

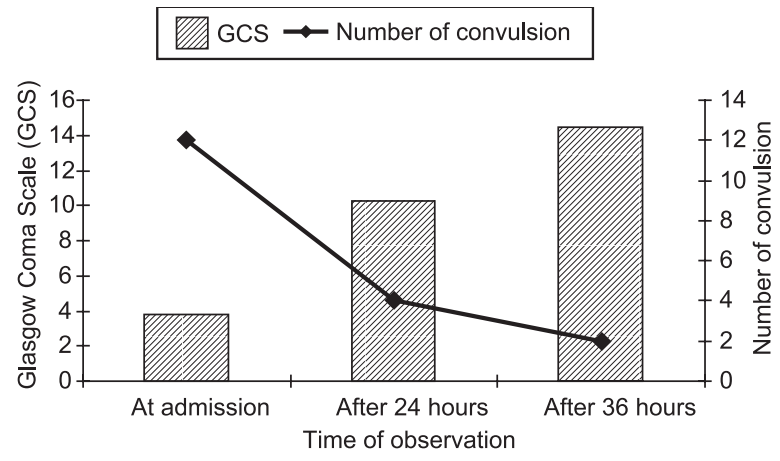

Fig.-1 


\section{Discussion:}

In normal pregnancy there is an early increase in cardiac output. Total body fluid is increased, serum protein fall and produce physiological oedema due to excess fluid in the interstitial space. In PE and eclampsia, plasma volume is usually reduced and colloidal osmotic pressure is reduced always. Buffering mechanisms are weak, peripheral oedema occurs and in severe cases there is oedema of brain and lungs. Crystalloids do not contain any large particles such as proteins, therefore do not stay within the blood vessels and can leak out of the plasma into the tissues. Crystalloid solutions move more quickly across the endothelium ${ }^{3}$. Colloid substances increase the colloid oncotic pressure (COP) and effectively move fluid from the interstitial compartment to the plasma compartment by pulling the fluid into the blood vessels therefore increasing blood volume. We managed these patients in eclampsia ward by fluid therapy with Ringers lactate solution one liter daily $0.9 \%$ sodium chloride, $25 \%$ glucose, $5 \%$ amino-acids along with hydrocortisone and in some cases nasogastric feedings in addition to other regular medications. We followed a systematic guideline and closely monitored those patients until the outcome. Fluid management required frequent clinical assessment and meticulous attention was given to make input and output chart and calculation of fluid balance. Glasgow Coma Scale (GCS), blood pressure and proteinuria was markedly improved within 36 hours in $80 \%$ cases. No death occured during our study. Only two patients were referred to intensive care unit. Patient with moderate or severe PE and eclampsia have a degree of glomerular endotheliosis. It resolved spontaneously in course of time. Hypovolumia was excluded as a cause of oliguria. Volume expansion in the presence of glomerular endotheliosis is hazardous. Frequent clinical assessment of oxygen saturation, hourly urine volume estimation and Glasgow Coma Scale (GCS) assessment were the tools of management. No invasive procedure was adopted. Regarding fluid and nutritional management, more research is needed to improve the management and our knowledge.

\section{Conclusion:}

Eclampsia is one of the most important cause of maternal death. To aware of its pathophysiology and acute management to reduce the maternal morbidity and mortality, which are still associated with this condition, should be known by all medical staff ${ }^{9}$. Protocols for the management should be available and reviewed. Input of adequate fluid and nutrition along with other required management and close monitoring can significantly reduce the mortality of unconscious eclamptic patients in resource poor settings where intensive care facility is limited. Similar studies are warranted to establish a universal adaptation guideline in all obstetrics units.

\section{Reference:}

1. S.C. Robson. High risk pregnancy managemetn option. Hypertension and renal disease in pregnancy; Chapter 15, 1995; BMJ Publishing Group: 166-185.

2. T. Engelhardt, F.M. Maclennan. Fluid management in pre-eclampsia; International journal of obstetrict anesthasia 1999; 8: 253-259.

3. C.P. Swainson, A.D. Cumming. Principle and Practise of Medicine. Disturbances in water, electrolyte and acid-base balance; 18th ed. Churchill Livingstone inc. 1999, 393-416.

4. Assali NsS, Vaughn DL. Blood volume in preeclampsia: Fantasy and reality. Am J object Gynecol 1977; 129: 355-359.

5. Cong KJ. hemodynamic surveillance in pregnancy induced hypertension during volume expansion therapy. Chung Hua Fu Chan Tsa Chih, 1992; 27: 5-8.

6. Duncan SL. Does volume expansion in preeclampsia help or hinder? Br.J Obstet Gynaecol 1989; 96: 631-633.

7. Fluid balance in pre eclampsia:what we know and wha we don't:Louise Ashelby et.al Progress in Obstetrics; Volume17 :Edited John Studd page 124-137.

8. Young PF, Leighton NA, Jones PW, Anthony J, Johanson RB. Fluid management in severe preeclampsia(VESPA): survey of members of ISSHP. Hypertens Pregnancy, 2000; 19(3): 249-59.

9. Duley L. Magnesium sulphate regimens for women with eclampsia: messages from the Collaborative Eclampsia Trial. Br J Obstet Gynaecol 1996; 103: 103-105

10. Osama Salha, James J walker. Modern management of eclampsia; Postgrad Med J 1999;75:78-82.

11. Duley L. Maternal mortality associated with hypertensive disorders of pregnancy in Africa, Asia, Latin America and the Caribbean. $\mathrm{Br} \mathrm{J}$ Obstet Gynaecol 1992; 99: 547-553.

12. Sibai BM. Eclampsia: VI. Maternal-perinatal outcome in 254 consecutive cases. Am J Obstet Gynecol 1990; 163: 1049-1055. 